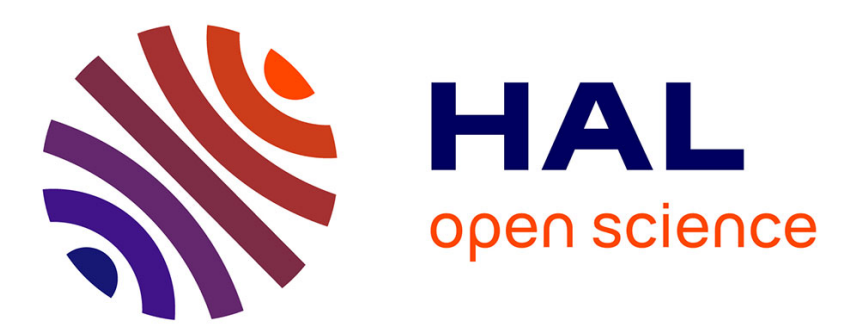

\title{
Enhancing Pinch-Drag-Flick Paradigm with Two New Gestures: Two-Finger-Tap for Tablets and Tap\&Tap for Smartphones
}

\author{
Alessio Bellino
}

\section{- To cite this version:}

Alessio Bellino. Enhancing Pinch-Drag-Flick Paradigm with Two New Gestures: Two-Finger-Tap for Tablets and Tap\&Tap for Smartphones. 15th Human-Computer Interaction (INTERACT), Sep 2015, Bamberg, Germany. pp.534-551, 10.1007/978-3-319-22698-9_37 . hal-01609390

\section{HAL Id: hal-01609390 https://hal.inria.fr/hal-01609390}

Submitted on 3 Oct 2017

HAL is a multi-disciplinary open access archive for the deposit and dissemination of scientific research documents, whether they are published or not. The documents may come from teaching and research institutions in France or abroad, or from public or private research centers.
L'archive ouverte pluridisciplinaire HAL, est destinée au dépôt et à la diffusion de documents scientifiques de niveau recherche, publiés ou non, émanant des établissements d'enseignement et de recherche français ou étrangers, des laboratoires publics ou privés. 


\title{
Enhancing Pinch-Drag-Flick Paradigm with Two New Gestures: Two-Finger-Tap for Tablets and Tap\&Tap for Smartphones
}

\author{
Alessio Bellino \\ University of Milano-Bicocca \\ Viale Sarca 336/14, Milano, Italy \\ bellinodisco. unimib.it
}

\begin{abstract}
The mobile versions of services such as Google Maps or Open Street Maps allow the exploration of maps on smartphones and tablets. The gestures used are the pinch to adjust the zoom level and the drag/flick to move the map. In this paper, two new gestures to adjust the zoom level of maps (but also of images and documents) are presented. Both gestures - with slight differences allow the identification of a target area to zoom, which is enlarged automatically up to cover the whole map container. The proposed gestures are added to the traditional ones (drag, pinch and flick) without any overlap. Therefore, users do not need to change their regular practices. They have just two more options to control the zoom level. One of the most relevant and appreciated advantages has to do with the gesture for smartphones (Tap\&Tap): this allows users to control the zoom level with just one hand. The traditional pinch gesture, instead, needs two hands. According to the test results on the new gestures in comparison with the traditional pinch, $30 \%$ of time is saved on tablets (Two-Finger-Tap gesture) whereas $14 \%$ on smartphones (Tap\&Tap gesture).
\end{abstract}

Keywords. zoom $\cdot$ map $\cdot$ touch $\cdot$ smartphone $\cdot$ tablet $\cdot$ gesture

\section{Introduction}

Currently, one of the most common activities performed by users in their mobile devices is the exploration of large $2 \mathrm{D}$ spaces such as maps, pictures and websites. In these devices, the use of zoom and drag is frequent because they usually have small screens. One of the most used multi-touch paradigms devoted to the $2 \mathrm{D}$ navigation is the Pinch-Drag-Flick because of its simplicity and effectiveness: pinch to zoom and drag-flick to pan. In spite of these advantages, the paradigm presents some drawbacks: fingers hinder the screen visualization [14] and the pinch is difficult to perform with just one hand when it comes to smartphones [13]. These disadvantages have fostered the development of several alternatives to navigate which, for instance, use different gestures [3] or extend the interaction to the side [12] or the back of the device [14]. 
In this paper, two new gestures are presented. These are added to the traditional paradigm Pinch-Drag-Flick without replacing it. Furthermore, they do not require specific hardware. One gesture is designed for tablets whereas the other for smartphones. Particularly, the latter allows the usage of smartphones with just one hand, unlike the traditional Pinch-to-Zoom in which both hands are required: one to hold the smartphone and the other to act on the screen. The new gestures were evaluated qualitatively and quantitatively with 18 users. In addition, a speed test between the traditional pinch and the new gestures was carried out with the aim to understand and evaluate the design qualities of the new gestures. A 'strict' comparison between the new gestures and the traditional ones does not make sense because there is no any overlap between them. The more traditional user, in fact, can use the new gestures in addition to the Pinch-Drag-Flick paradigm. Any comparison would be probably useless, unless users are not willing to change their regular practices. Changing users' behavior is a more complex and hard challenge than any speed test.

\section{Related work}

The Pinch-Drag-Flick paradigm is used frequently nowadays, therefore, no explanation would be required. Nevertheless, since an integration to the aforementioned paradigm has been designed, some details are provided when the new gestures are explained (3.3). This section, instead, is focused on spatial input-based interactions and some alternatives to the traditional Pinch-Drag-Flick paradigm. In [5], one of the first spatial input-based technique used on mobile devices is presented. This technique is driven by the see-through interfaces [2] metaphor, in which the interaction with the real world is performed by moving the device around. A recent study (2014) [13] presents a similar approach: moving the device leftwards, rightwards, upwards and downwards the pan is performed; moving the device away or bringing it closer, the zoom out or zoom in is performed respectively. The performances of spatial input interaction shown in [13] regarding time of zoom on 2D spaces (such as maps) are better than pinch. Their research is focused on smartphones and tablets as in the present study. However, the devices used in [13] were modified with infrared led lights. Through these, IR-cameras in the environment detect the movements of the device. Smartphones and tablets require, thus, modifications and additional hardware to work. Yet, the gestures proposed in this paper do not need neither modifications in the devices nor the use of ad hoc hardware. Another kind of zoom - which also exploits the device movements but it cannot be considered a real spatial input - is the Tilt-toZoom [7], which allows the adjustment of the zoom tilting the smartphone detecting its movements through the accelerometer. The spatial input approaches, unlike the touch input (of which the Pinch-Drag-Flick is the main one), require the movement of the device around the physical space. Therefore, the movements increase significantly (3D physical space versus 2D small screen) and different kinds of movements are required (arm versus hand/fingers) [13].

The aim of this research is not to debate about which the best approach is. Some studies have proven that there are not clear advantages for the spatial input-based ap- 
proach ([6], [10]). Other recent studies have revealed that there are some improvements, and combining spatial input and touch input may be useful (i.e. the first one for navigation whereas the second one for the selection) [13]. From a more social viewpoint, the use of the spatial input could be amusing sometimes. There could be other instances, instead, in which users do not feel like using the spatial input and the Pinch-Drag-Flick paradigm is more appropriate (e.g., when users prefer to be in a fixed position). Anyways, the Pinch-Drag-Flick paradigm is deep-rooted in the market and abandoning it is as difficult as changing the users' practices (think of Dvorak keyboard). Coming back to touch interaction, some alternative paradigms are Slider, CycloStar [9] and Fat Thumb [3]. The first one, coming from desktop computers, is not common in touch devices. However, it allows the zoom adjustment by moving the slider upwards or downwards. The second [9] works by moving the finger in circles on the device screen: clockwise to zoom in and anticlockwise to zoom out. The third [3] lets users adjust the zoom by means of the contact area of their thumbs with the screen: with slight pressure, the contact area is small and the pan is allowed by moving the finger. On the contrary, with high pressure, the contact area is larger and the zoom adjustment is allowed. In this case, the zoom speed depends on the size of the contact area of the thumb. All these paradigms allow the zoom usage with just one hand. In [3] a comparison among Tilt-to-zoom [7], Slider, CycloStar [9] and Fat Thumb [3] was made and the latter is the faster.

Some commercial software for desktop computers implement another way of zoom quite interesting for this research: identifying an area by means of selection, it will be enlarged automatically up to cover the dimension of the window container. This zoom is called in different ways: Marquee Zoom in Adobe Acrobat $\mathrm{X}^{1}$ and Zoom Window in Autodesk AutoCAD ${ }^{2}$. Moreover, a patent that uses a similar zoom principle was registered [4]. The two new gestures presented in this paper use an approach similar to this kind of zoom for desktops.

\section{Gesture definition}

\subsection{Two-Finger-Tap for tablets}

The gestures designed for tablets, namely Two-Finger-Tap, let users zoom in by tapping the screen with two fingers suitably spaced.

Fig. 1 displays the whole zoom process: in the first step, users tap with two fingers the target area to be zoomed. In the second step, the algorithm identifies the area comprehended between the fingers (ideally, it may be a circle), which is enlarged automatically up to cover the map container as shown in step 3 . The process is iterative:

1 http://help.adobe.com/en_US/acrobat/X/pro/using/WS58a04a822e3e50102bd6151097941 95ff-7aec.w.html

2 http://knowledge.autodesk.com/support/autocad/learn-explore/caas/CloudHelp/cloudhelp/ 2015/ENU/AutoCAD-Core/files/GUID-66E7DB72-B2A7-4166-9970-9E19CC06F739htm.html 
after the first zoom, the user can continue to zoom in starting again from the first step. In order to zoom out, the traditional pinch-out has to be used as shown in Fig. 2.

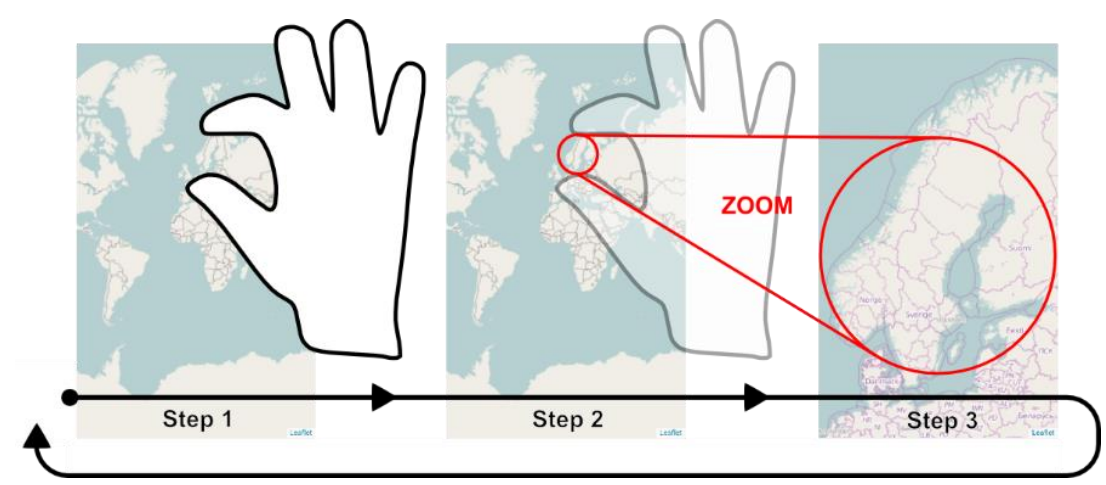

Fig. 1. Two-Finger-Tap gesture: zoom in.

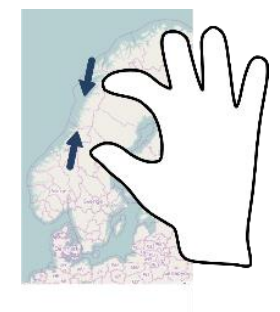

Fig. 2. Two-Finger-Tap gesture: zoom out.

\subsection{Tap\&Tap for smartphones}

The gesture was designed to be used holding the smartphone with just one hand interacting with the thumb. This choice was made to favor the users who prefer to use the smartphone with just one hand (around 49\%) [8]. The traditional pinch, instead, compels users to employ both hands: one to hold the smartphone and the other to interact with the map on the screen.

The gesture, namely Tap\&Tap, let users zoom in by touching in fast sequences two different points of the map. As in the previous process, the target area identified between the two points is enlarged automatically up to cover the map container (Fig. 3). The one-hand usage is also kept to zoom out: users have to scroll the thumb from the left edge of the screen as shown in Fig. 4.

\subsection{Compatibility: new gestures and Pinch-Drag-Flick}

The Two-Finger-Tap gesture comes into conflict with the gesture set of some commercial software for map navigation (i.e. Google Maps or Apple Maps): in their implementation, a tap with two fingers is used to zoom out one level in opposition to the traditional double tap (which is used to zoom in one level). At any rate, that gesture is 
unknown by users (none of the users who took the test was acquainted with it) and seems not to be that useful because it is similar to the traditional pinch-out (beyond the fact that two hands are needed in smartphones). Other frameworks for map navigation (e.g. Open Street Maps or Leaflet), in fact, do not implement that gesture. Moreover, neither Google mentions that gesture in the tips\&trick official area ${ }^{3}$. For these reasons, that gesture is not taken into account in the following discussion.

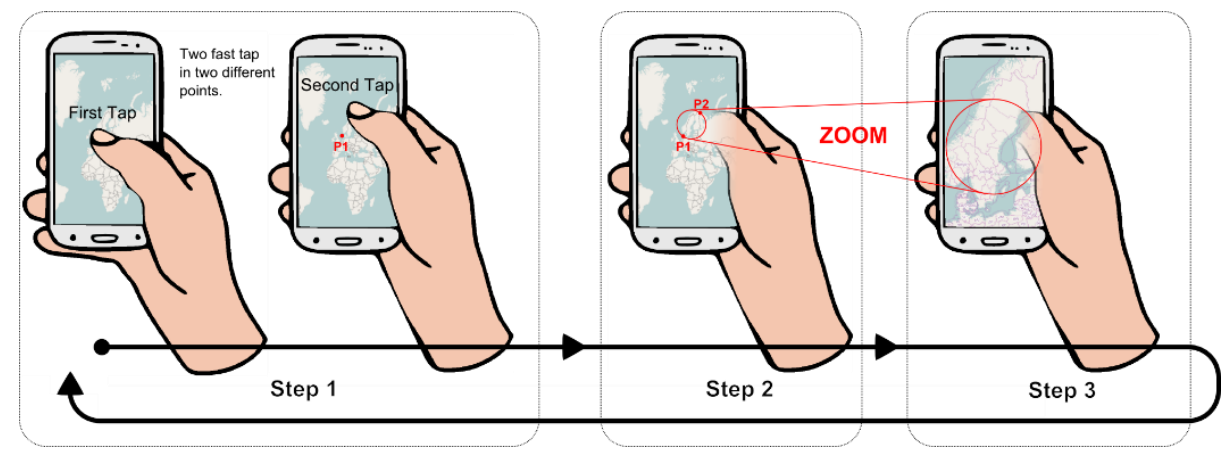

Fig. 3. Tap\&Tap gesture: zoom in.

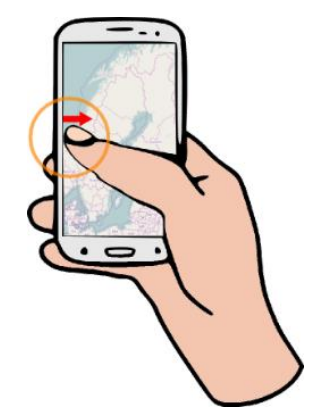

Fig. 4. Tap\&Tap gesture: zoom out.

In order to understand the mechanisms through which the integration with the Pinch-Drag-Flick paradigm is designed without overlapping, a review of the traditional gestures is presented here:

1. Pinch: it allows the zoom adjustment by touching the screen with two fingers expanding/shrinking them (Fig. 5 line A).

2. Drag: it allows the pan of the map by moving the finger in all directions (Fig. 5 line B).

3. Double-Tap: it allows the zoom in with just one hand by using a double tap. The zoom starts from the point tapped (Fig. 5 line C).

4. DoubleTap-and-Hold + Vertical-Drag: it allows the zoom adjustment with just one hand by tapping twice, holding the screen the second time and moving the

3 https://support.google.com/gmm/answer/3273126 
finger upwards (zoom out) or downwards (zoom in) (Fig. 5 line D). This gesture was introduced by Google Maps.

\begin{tabular}{|c|c|c|c|c|c|c|}
\hline Touch events & Touch start 1 & Touch move 1 & Touch end 1 & Touch start 2 & Touch move 2 & Touch end 2 \\
\hline A. Pinch & (2) & * & (2) & & & - \\
\hline B. Drag & (2) & & (2) & & & \\
\hline C. Double-tap & (2) & NO MOVEMENT & (2) & (2) & NO MOVEMENT & (2) \\
\hline D. Double-tap and hold + Drag & (2) & NO MOVEMENT & (2) & (2) & ழุ & (2) \\
\hline E. Tap\&Tap & (2) & NO MOVEMENT & (2) & (2) & NO MOVEMENT & (2) * \\
\hline F. Two-Finger-Tap & (2) & NO MOVEMENT & (2) & & & \\
\hline
\end{tabular}

Fig. 5. Touch detection algorithm. P1, P2, P3 and P4 indicate four different touch points on the screen. The red star indicates the moment in which each gesture is detected. Touch start indicates the moment in which fingers touch the screen; touch move indicates the moment in which fingers are moving on the screen; touch end indicates the moment in which fingers release the screen. Some gestures can be detected only repeating the process start/move/end. For example, the required sequence for 'DoubleTap-and-hold + Drag' is 'touch start', 'no movement' and 'touch end' (first tap) and continuing 'touch start', 'a movement' and 'touch end' (second taphold + drag [-and release]). In this example, the gesture is detected (it starts to work) when 'touch move 2' occurs.

The proposed gestures look like some of the aforementioned gestures. However, they are quite different and their usage is not ambiguous. In particular, the Tap\&Tap gesture for smartphones is similar to the Double-Tap. Anyway, the Tap\&Tap occurs only when the second tap touches a different point from the previous one (Fig. 5 line E), whereas the Double-Tap occurs only when the two taps touch the same point (Fig. 5 line C). Going on, the Two-Finger-Tap gesture is similar to the pinch. Anyway, the Two-Finger-Tap occurs only when fingers remain fixed on the screen and are released immediately after (Fig. 5 line F), whereas the pinch occurs only when fingers move on the screen expanding or shrinking (Fig. 5 line A, see touch move 1). The entire gesture set in Fig. 5, without producing any overlap, lets users increase their possibilities to choose the most appropriate gesture in any conditions (tablets, smartphones, one-hand usage, two-hands usage). In this discussion, the Tap\&Tap zoom-out gesture (Fig. 4) was not taken into account: since only the edge of the screen is involved (Bezel Swipe [11]), there is not any overlap.

Finally, the conceptual differences between the pinch and the new gestures are clarified in order to better understand their nature. The pinch is 'incremental': the zoom adjustment works continuously following the finger movements. The new ges- 
tures are 'fit-area-to-container': the identified area comprehended between two points of the map is automatically enlarged up to cover the map container (like Adobe Acrobat $\mathrm{X}$ and Autodesk AutoCAD previously mentioned in the related work).

\section{User's evaluation}

Although one gesture was designed for tablets and the other for smartphones, their evaluation in each device was considered. Nonetheless, preliminary tests have revealed that the Tap\&Tap - originally designed for smartphones - is not suitable for tablets. Even though (i) tablets are usually used with two hands and the interaction occurs with the index finger, the thumb is near the screen, ready to be used (Fig. 6). Therefore, the Two-Finger-Tap makes sense, unlike the Tap\&Tap.

Moreover, (ii) the distance between the two rapid taps in sequence - required by the Tap\&Tap - could be ample due to screen dimensions (Fig. 7). That is why the Tap\&Tap was not evaluated on tablets. On the contrary, the Two-Finger-Tap - originally developed for tablets - was evaluated also on smartphones.

Table 1 shows the three evaluation stages.

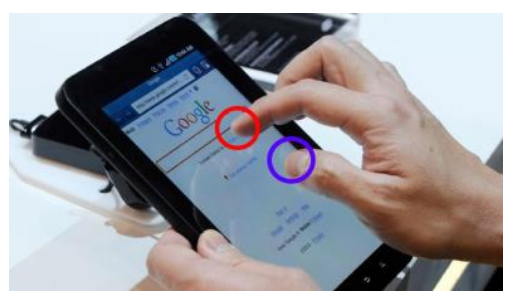

Fig. 6. Even if the index finger is normally used for interaction on tablets, the thumb is usually near the screen, ready to be used.

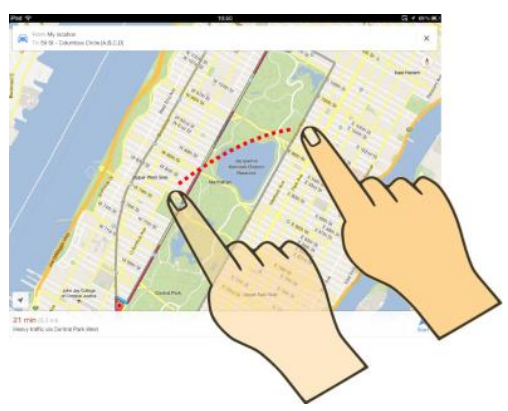

Fig. 7. The distance between taps could be too long to make the interaction usable.

\subsection{Representative population}

The evaluations involved a 22-42 age group of 18 users (one of them was a woman) split in five age brackets (Fig. 8A).

Table 1. Stages for gesture evaluation through questionnaire.

\begin{tabular}{|c|c|c|}
\hline Tablet & \multicolumn{2}{|c|}{ Smartphone } \\
\hline Two-Finger-Tap (stage one) & Tap\&Tap (stage two) & Two-Finger-Tap (stage three) \\
\hline
\end{tabular}


All users asserted they use touch devices regularly (Fig. 8C). 10 out of 18 users asserted they use the smartphone with just one hand regularly, whereas the others with two hands or sometimes with one hand and sometimes with two hands (Fig. 8B). 10 users asserted they could need to use maps with one hand frequently, seven users do rarely and one user never had the need of using maps with just one hand (Fig. 8D). Four users asserted they use maps daily, 10 users do weekly, three users do monthly and one user sometimes a year (Fig. 8E).

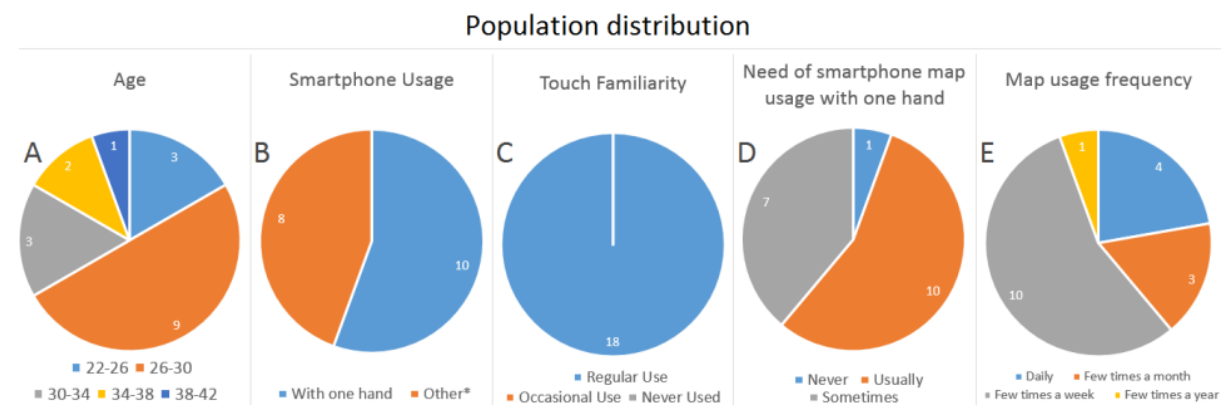

Fig. 8. Representative population. *Other indicates sometimes with one hand and sometimes with both hands.

\subsection{Hardware and software employed}

In order to carry out the tests, a smartphone Motorola Moto G (4-inch with Android OS) and a tabletop Lenovo Flex 2-15 (15-inch with Windows 8 OS) were used. The smartphone was used in portrait mode whereas the tabletop in landscape mode. The prototype works in both devices in the same way and was developed in HTML5, JavaScript and CSS3 redesigning Leaflet ${ }^{4}$, an open source library for maps management optimized for mobiles (maps are provided from Open Street Maps). Both devices display the prototype at full screen through Google Chrome browser (in the respective versions for Android and Windows 8).

Further details on the implementation are presented in [1].

\subsection{The test procedure}

The tests were carried out in a lab of the university. First of all, users watched an introductory video in which gestures were described while working. After that, the first stage was the test of the Two-Finger-Tap on the tabletop, followed by the test of the Tap\&Tap (second stage) and the Two-Finger-Tap (third stage) on the smartphone (see Table 1). For each stage, the following tasks were carried out: starting from the whole world, it was requested to: 1) zoom in up to Sardinia (Italian island) 2) go back, 3) zoom in up to Paris, 4) go back, 5) zoom in up to Madrid, 6) go back, 7) zoom in up

4 http://leafletjs.com 
to the United Kingdom, 8) go back, 9) zoom in up to a city chosen by the user and 10) go back.

At the end of the three stages, answering a questionnaire was requested.

\subsection{The questionnaire}

The questionnaire is composed by four sections. In the first, the users' information was gathered (see Fig. 8). In the following three sections (which correspond to the three stages), it was asked users to evaluate the Two-Finger-Tap on tablets (first stage), the Tap\&Tap on smartphones (second stage) and, finally, the Two-Finger-Tap on smartphones (third stage) using a 6-point Likert scale (Fig. 9).

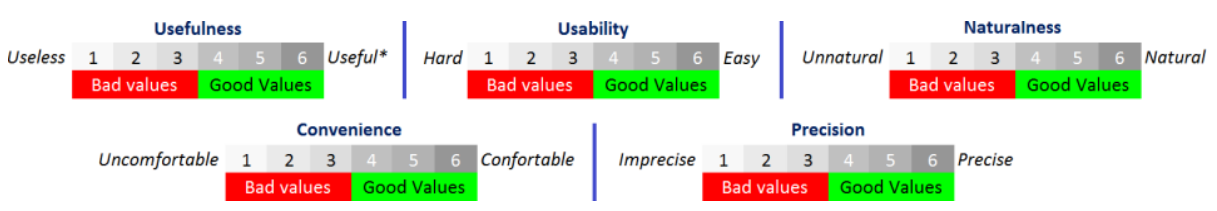

Fig. 9. Section of the questionnaire regarding the evaluation given for each gesture. *The full sentence for useful is: "Useful, the gesture is a good alternative to the traditional pinch".

\subsection{Time measurement}

At the end of the questionnaire (to avoid possible biases), it was asked users to take a speed test (composed by 20 tasks) in order to measure the execution times of zoom with the new gestures in comparison with the traditional pinch. The software calculated the execution times automatically.

\subsection{Correlation analysis}

A correlation analysis was carried out using Spearman's rho test.

\subsection{Qualitative analysis}

After the time measurements, it was asked users to provide an opinion regarding the new gestures promoting a discussion.

\section{$5 \quad$ Experimental results}

\subsection{Questionnaire}

A binomial test was conducted on the data gathered from the Likert scale for each stage. Values from 1 to 3 were considered negative whereas values from 3 to 6 were considered positive (see Fig. 9). 
The binomial test results on Two-Finger-Tap gesture for tablets (first stage) show clear positive tendencies $(\mathrm{p}<0.05)$ for usability (proportion $.17 \mathrm{vs.} 87, \mathrm{p}=0.008$ ), naturalness (proportion .17 vs. 83, $\mathrm{p}=0.008$ ), convenience (proportion .22 vs. 78, $\mathrm{p}=0.031$ ), and usefulness (proportion .22 vs. $78, \mathrm{p}=0.031$ ). Regarding precision (proportion .28 vs. $72 \mathrm{p}=0.096$ ), it is not possible to establish any clear positive tendency ( $p>0.05)$. A summary of the results is displayed in Fig. 10 through box-plots with mean (red) and mode (blue).

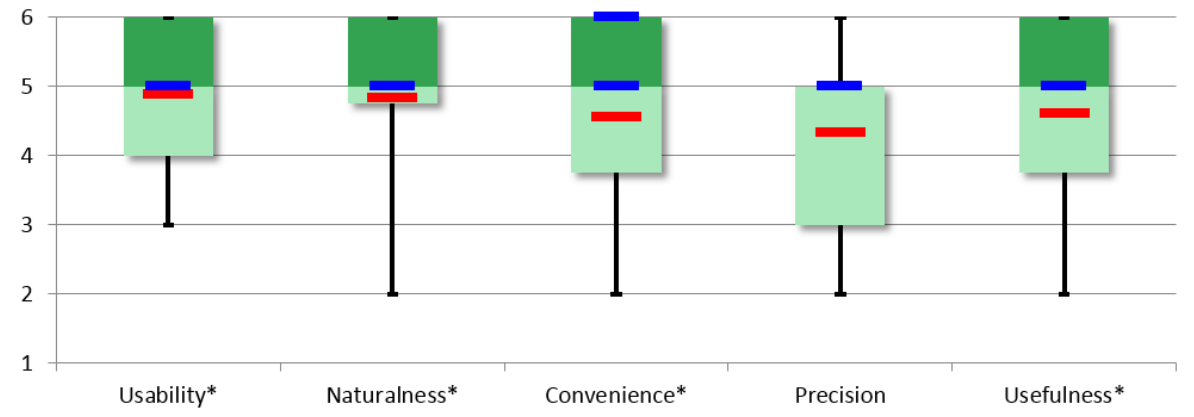

Fig. 10. Two-Finger-Tap evaluations on tablets.

The binomial test results on Tap\&Tap gesture for smartphones (second stage) show clear positive tendencies $(\mathrm{p}<0.05)$ for usability (proportion .00 vs 1.00 , $\mathrm{p}<0.001$ ), convenience (proportion .06 vs. 94, $\mathrm{p}<0.001$ ), precision (proportion $.17 \mathrm{vs}$ $.83, \mathrm{p}=0.008$ ) and usefulness (proportion .11 vs. $89, \mathrm{p}=0.001$ ). Regarding naturalness (proportion .28 vs. $72 \mathrm{p}=0.096$ ), it is not possible to establish any clear positive tendency $(p>0.05)$. A summary of the results is displayed in Fig. 11 through box-plots with mean (red) and mode (blue).

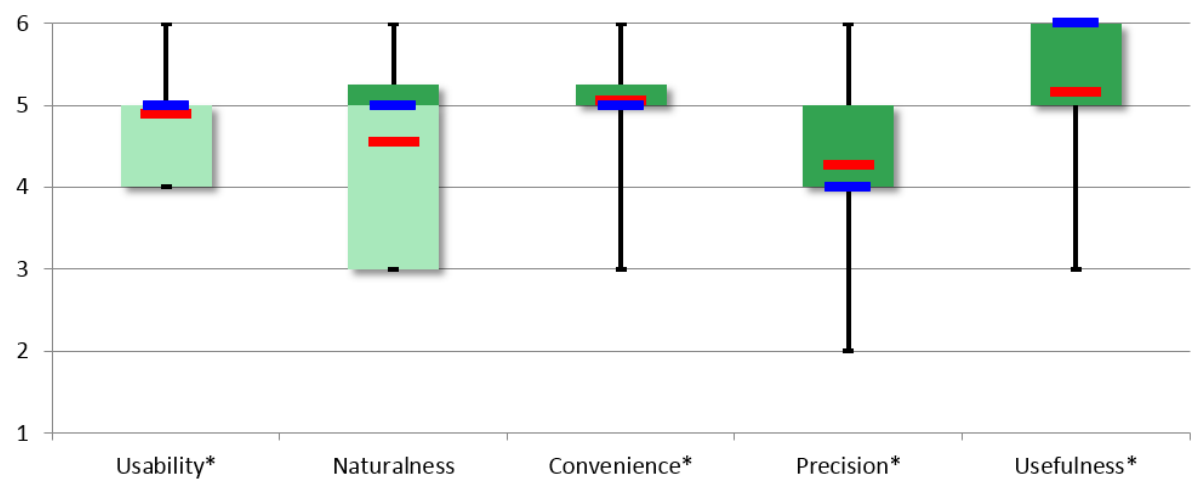

Fig. 11. Tap\&Tap evaluations on smartphones.

The binomial test results on Two-Finger-Tap for smartphones (third stage) do not show clear positive tendencies in any of the variables $(\mathrm{p}>0.05)$ : usability (proportion .44 vs. $56, p=0.815$ ), naturalness (proportion .44 vs. $56, p=0.815$ ), convenience (pro- 
portion .61 vs. $39, \mathrm{p}=0.481$ ), precision (proportion .50 vs. $50, \mathrm{p}=1$ ), and usefulness (proportion .50 vs. $50, \mathrm{p}=1$ ). A summary of the results is displayed in Fig. 12 through box-plots with mean (red) and mode (blue).

In the light of the results previously presented, the Two-Finger-Tap for tablets and the Tap\&Tap for smartphones are appreciated by users. Moreover, as expected, they are considered as a good alternative to the traditional pinch. For the Two-Finger-Tap for smartphones, such negative results were not expected. Anyway, evaluations lower than the Tap\&Tap were predictable.

\subsection{Zoom speed measurement}

After the questionnaire, each user performed 20 tasks (split in five stages, two for tablets and three for smartphones, see Fig. 13) devoted to the time measurements of the pinch in comparison with the new gestures.

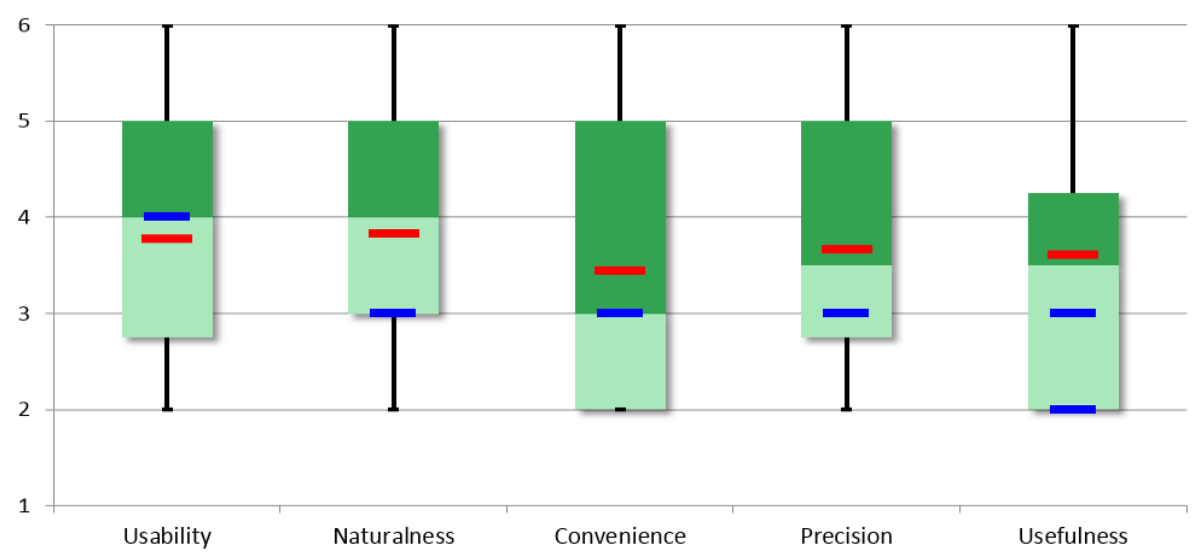

Fig. 12. Two-Finger-Tap evaluations on smartphones.

For each stage, the tasks executed by users consisted in zooming inside a rectangle that identified a fragment of the world established by the experimenter. In any case, the zoom started from the whole world (Fig. 14, left). The first task consists in zooming inside the rectangle (53122 km2) that comprehends Sicily (Fig. 14, task 1) an Italian island. The second task consists in zooming inside the rectangle $(4.5 \mathrm{~km} 2)$ that comprehends Bicocca (Fig. 14, task 2), a neighborhood of Milan. The third task consists in zooming inside the rectangle $(121820 \mathrm{~km} 2)$ that comprehends the eastern part of the Denmark (Fig. 14, task 3). The fourth task consists in zooming inside the rectangle $(2361 \mathrm{~km} 2)$ that comprehends Berlin (Fig. 14, task 4). The average time on the 18 users was calculated both for smartphones and tablets for each place and every gesture (gray rows in Fig. 13). The total time, for each gesture, was calculated summing the execution times of each place (the red row in Fig. 13).

Since the new gestures do not hinder the usage of the traditional ones, a comparison between the execution times of the new gestures with the traditional pinch, would be probably useless. Therefore, the aim of this comparison is to understand better how 
the new gestures work. Since some of the data was not normally distributed (ShapiroWink test), the non-parametric Wilcoxon signed rank test is used to evaluate whether two distributions are statistically different.

The average zoom speed with Two-Finger-Tap on tablets is $15334 \mathrm{~ms}$ ( $\mathrm{SD}=4995)$ whereas with the traditional pinch is $22186 \mathrm{~ms}$ ( $\mathrm{SD}=4994)$. The new gesture TwoFinger-Tap on tablets saves $30 \%$ of time. The difference between distributions is significant $(\mathrm{Z}=-3.680 ; \mathrm{p}<0.001)$. The average zoom speed with Tap\&Tap on smartphones is $17414 \mathrm{~ms}(\mathrm{SD}=2595)$ whereas with the traditional pinch is $20482 \mathrm{~ms}$ $(\mathrm{SD}=2721)$. The new gesture Tap\&Tap on smartphones saves $15 \%$ of time. The difference between distributions is significant $(\mathrm{Z}=-3.462 ; \mathrm{p}=0.001)$. The average zoom speed with Two-Finger-Tap on smartphones is $16960 \mathrm{~ms}(\mathrm{SD}=3092)$ whereas with the traditional pinch is $20482 \mathrm{~ms}$ ( $\mathrm{SD}=2721)$. The new gesture Two-Finger-Tap on smartphones saves $17 \%$ of time (even though this gesture is not that appreciated by users as shown in Fig. 12). The difference between distributions is significant $(\mathrm{Z}=-$ 3.506; $\mathrm{p}<0.001)$.

See Fig. 13 for the average of the execution times, standard deviations and comparisons among places. Significant differences between distributions are marked with an asterisk.

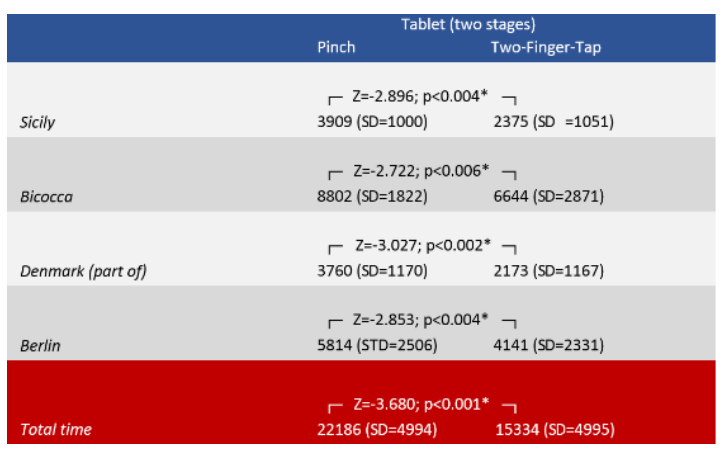

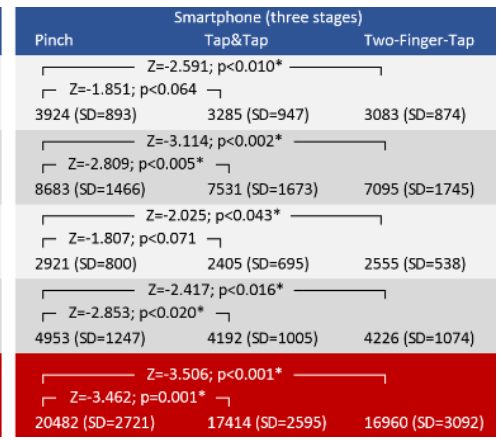

Fig. 13. Stages of tests for speed evaluations (average in milliseconds on 18 users with its standard deviation). The distribution of each new gesture (Two-Finger-Tap and Tap\&Tap) in each device was compared with the ones of the traditional pinch evaluating, using the Wilcoxon signed rank test, whether they are statistically different.

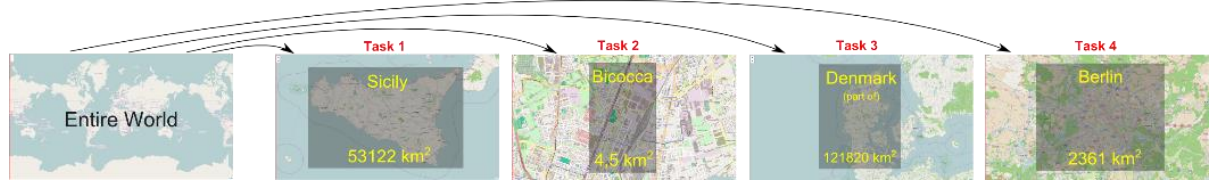

Fig. 14. Tasks for speed tests.

Discussion. Little relevance is given to the speed tests results in this study. Actually, on tablets, the Two-Finger-Tap has a moderate advantage in comparison with the pinch. Regarding smartphones, the $15 \%$ of time saved given by the Tap\&Tap does not seem to be that relevant for users. Taking into account the absolute times shown in 
Fig. 13, in the best of the cases, an advantage of around 2 seconds is given by the new gestures. These advantages seem to be irrelevant in the regular use of maps. At any rate, there are other relevant factors to highlight; besides the little advantages in terms of execution time, the new gestures are considered useful by users because they allow the zoom adjustment in an alternative and effective way. In particular, the advantages of the Tap\&Tap are related to the employment of just one hand with execution times comparable with the traditional pinch.

Although the execution time analysis is not used to spread out inexistent advantages, it could be useful to understand more about the new gestures. Actually, an improvement of execution times directly proportional with the depth of zoom was expected. This hypothesis was rejected by the results shown in Fig. 13. In particular, the task in which the highest depth of zoom is needed (Bicocca, $4.5 \mathrm{~km} 2$ ) does not display better results than the task in which the lowest depth of zoom is needed (Denmark, $121820 \mathrm{~km} 2$ ). For example, the Two-Finger-Tap on tablets saves $24 \%$ of time on the task of Bicocca and $42 \%$ on the task of Denmark. These results are contradictory considering the initial expectations. An explanation is given by a further time execution analysis.

\subsection{Further time execution analysis}

The execution times previously presented were calculated considering the sum of the times in three different phases:

1. The zoom phase occurs when the map is enlarged or stretched (Fig. 15A).

2. The drag ${ }^{5}$ phase occurs when the map is moved centering the target area to zoom (Fig. 15B).

3. The planning phase occurs when users (release the finger from the screen - Fig. $15 \mathrm{C}$ - and) plan the next action (another zoom or drag phase). The planning usually also includes the time needed by the system to load new tiles ${ }^{6}$. In fact, if the map is not refreshed, users cannot plan the next action easily (unless they go blindly, remembering the place locations even when the map is not loaded yet).
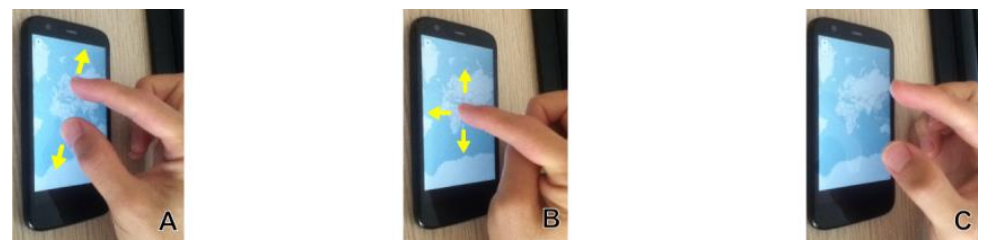

Fig. 15. Three zoom phases: zoom (A), drag (B) and planning (C).

5 The more skilled users who use the traditional pinch are usually able to merge the zoom and the drag phase. In other words, they are able to zoom and move the map at the same time. Anyway, it is not always convenient and/or possible when the target area to zoom is near the border of the screen. Therefore, also for the more skilled users, some movements of the map (drags) are essential.

6 http://wiki.openstreetmap.org/wiki/Tiles 
This section shows how different gestures employ time considering the aforementioned phases. In general, both the new gestures let users save time on the zoom and drag phases whereas the execution time of the planning phase increases (unlike the expectations). Fig. 16 shows that every difference among zoom, drag and planning time for each gesture is significant $(\mathrm{p}<0.05)$ : also in this case, the non-parametric Wilcoxon signed rank test is used to evaluate whether two distributions are statistically different.

To begin with, the results on smartphone are presented. The average time of drag on the pinch is $2221 \mathrm{~ms}(\mathrm{SD}=955)$, on the Tap\&Tap is $1140 \mathrm{~ms}(\mathrm{SD}=777)$, whereas on the Two-Finger-Tap is $821 \mathrm{~ms}(\mathrm{SD}=510)$. The average time of zoom on the pinch is $12750 \mathrm{~ms}$ ( $\mathrm{SD}=1824)$, on the Tap\&Tap is $4444 \mathrm{~ms}(\mathrm{SD}=1588)$, whereas on the TwoFinger-Tap is $8362 \mathrm{~ms}(\mathrm{SD}=1569)$. The average time of planning on the pinch is $5510 \mathrm{~ms}$ ( $\mathrm{SD}=2191)$, on the Tap\&Tap is $11829 \mathrm{~ms}(\mathrm{SD}=2628)$, whereas on the TwoFinger-Tap is $7776 \mathrm{~ms}(\mathrm{SD}=2692)$.

Then, the results on tablet are presented. The average time of drag on the pinch is $1497 \mathrm{~ms}(\mathrm{SD}=1797)$, whereas on the Two-Finger-Tap is $381 \mathrm{~ms}$ ( $\mathrm{SD}=468)$. The average time of zoom on the pinch is $11722 \mathrm{~ms}(\mathrm{SD}=2220)$, whereas on the Two fingerTap is $4416 \mathrm{~ms}$ ( $\mathrm{SD}=1256)$. The average time of planning on the pinch is $8965 \mathrm{~ms}$ ( $\mathrm{SD}=3016)$, whereas on the Two-Finger-Tap is $10536 \mathrm{~ms}(\mathrm{SD}=4133)$.

Fig. 16 shows the histograms of the total execution times (the same of the red row in Fig. 13) for each gesture: the drag phase is colored in blue, the zoom phase is colored in green and the planning phase is colored in yellow.

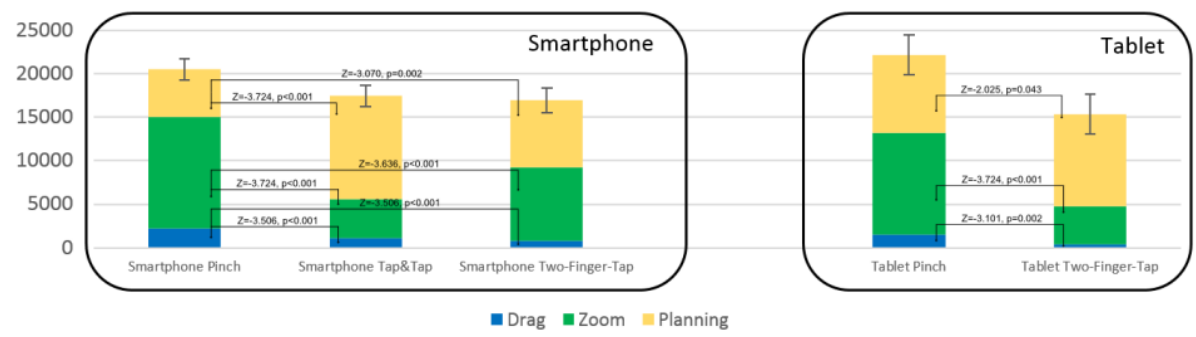

Fig. 16. Comparison among the execution time of each gesture phase with Wilcoxon signed rank test results: drag in blue, zoom in green and planning in yellow. Error bars denote standard deviations $(95 \% \mathrm{CI})$ on the total time (drag + zoom + planning).

Discussion. With the new gestures, in comparison with the pinch, it was expected: 1) a strong reduction of the drag time, 2) a strong reduction of the zoom time (because it is automatic, performed by the prototype using a fast animation), 3) a substantial equality of the planning time. The drag time of the new gestures, on every device, decrease as expected (-48\% for Tap\&Tap for smartphones, $-63 \%$ for Two-Finger-Tap for smartphones and $-74 \%$ for Two-Finger-Tap for tablets). The zoom time of the new gestures, on every device, decreased as expected $(-65 \%$ for Tap\&Tap for smartphones,

$-34 \%$ for Two-Finger-Tap for smartphones and $-62 \%$ for Two-Finger-Tap for tablets). 
The planning time of the new gestures, on every device, had a significant increase $(+114 \%$ for Tap\&Tap for smartphones, $+41 \%$ for Two-Finger-Tap for smartphones and $+17 \%$ for Two-Finger-Tap for tablets). Reflecting on this increase, the hypothesis is that users get lost due to the automatic zoom of the new gestures. Thus, it takes them more time to understand 1) where they are and 2) what to do (planning the next action). In the traditional pinch, instead, the zoom follows the movement of the fingers guiding the user's attention, so that users are more aware of what is going on. Hence, planning time is lower. In order to test the hypothesis, the variation of the execution times considering the three phases (drag, zoom, and planning) was examined according to different depths of zoom. Accordingly, it can be confirmed that the increase of the planning time with the new gestures is the reason why the save of time in percentage terms - on the task on Bicocca (higher zoom) is lower than the one on Denmark (lower zoom) as noted in the previous section. Zooming in on Bicocca, in fact, requires different zoom sequences; so that, the planning time increases, probably, because users feel more disoriented due to the repeated zoom sequences. This way, as shown in Fig. 17, the advantages related to the higher zoom speed of the new gestures are partially lost due to the lower planning speed. Fig. 17 shows the example of tablets but the same also occurs with the new gestures for smartphones. In any case, the planning time is likely to decrease if users get used to working with the new gestures.
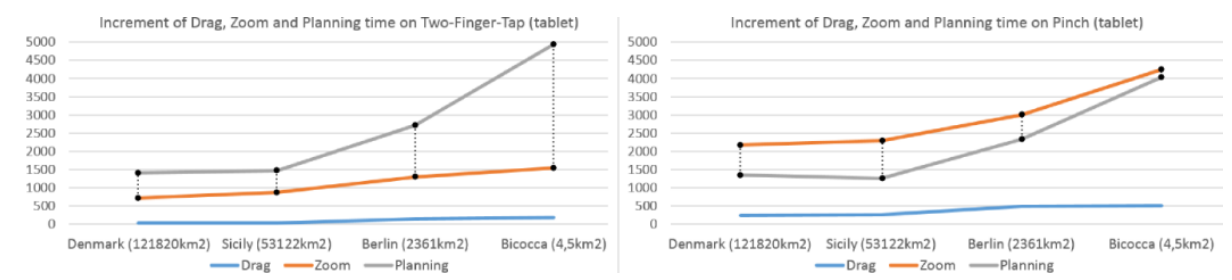

Fig. 17. Drag, Zoom and Planning time comparison according to Two-Finger-Tap and Pinch on tablets. In the graphic about the Two-Finger-Tap gesture (left), the increase of the gap between zoom and planning (black dotted line) is directly proportional to the zoom level (having a low zoom time but a high planning time on the Bicocca task). On the contrary, in the graphic about the traditional pinch (right) the gap is substantially constant.

\subsection{Correlation analysis}

41 correlations were found using Spearman's rho test. The more interesting ones are presented in this section. Correlations regarding the Two-Finger-Tap for smartphones are not put forward because it was not appreciated by users and the available space is not enough.

Age and need of using maps with just one hand are moderately inverse correlated $(\mathrm{r}=-590, \mathrm{p}=.010)$, therefore, the youngest users show a tendency towards the usage of maps with just one hand. In addition, frequency of usage of maps is slightly correlated with the need of using maps with just one hand $(\mathrm{r}=475, \mathrm{p}=.046)$. This means that providing a gesture that can be used with just one hand could be useful for those who use maps very often. 
As expected, the usefulness of the gesture Tap\&Tap is moderately correlated with the need of using maps with just one hand $(r=.619, p=.006)$. The usefulness of the same gesture is also moderately correlated with the frequency of usage of maps $(\mathrm{r}=.603, \mathrm{p}=.008)$. If this gesture were implemented in commercial software, people would probably use it.

The execution time of Tap\&Tap on smartphones is moderately correlated with their convenience $(\mathrm{r}=.580, \mathrm{p}=.012)$. This is interesting because the users took the speed test only after the questionnaire. This means that the (fast) users thought they were fast also before the direct comparison with the pinch.

Other two noteworthy correlations are: 1) the execution time of the pinch gesture on smartphones and the execution time of the Tap\&Tap gestures on smartphones are moderately correlated $(\mathrm{r}=.507, \mathrm{p}=.032) ; 2)$ the execution time of the pinch gesture on tablets and the execution time of the Two-Finger-Tap gestures on tablets are moderately correlated $(\mathrm{r}=.697, \mathrm{p}=.001)$. These two correlations mean that fast (or slow) people with the traditional pinch gesture are fast (or slow) with the new gestures too. Thus, the new gestures are practical to use. In fact, also in the case of a strong experience with the pinch (fast usage), the introduction of the new gestures seem to be favorable (people who are fast with the pinch are fast with the new gestures too).

Another moderated correlation $(\mathrm{r}=.692, \mathrm{p}=.001)$ was found between the execution time of the Two-Finger-Tap on tablets and the execution time of the Tap\&Tap on smartphones.

Several significant correlations were also found between questionnaire variables of the same gesture: they were expected, they do not seem to be very interesting and other details are not shown.

\subsection{Qualitative analysis}

After the speed test, users have provided their opinions regarding the new gestures. The most significant ones are translated and discussed here.

P. "If I had taken the questionnaire after the speed test, I would have changed a lot of answers. I understood the pinch is an old and uncomfortable gesture. The Tap\&Tap on smartphones is more comfortable and I would really like the gesture to be implemented in existent applications". This user was so enthusiastic that he was willing to look for other participants for the study. Besides that, his opinion is interesting because the evaluations on the questionnaire, probably, would be better after the speed test (in which the execution time of the pinch was compared with the one of the new gestures). Moreover, this comment confirms that doing the speed test after the questionnaire was a good choice in order to avoid bias.

C. "I had the need to zoom with one hand and I noted that Google Maps was the first which implemented a similar possibility. Nevertheless, your gesture is more precise and fast". This comment proves that the need to zoom with just one hand is very relevant for some users (he had looked for this option). In addition, after the test, the user said to a friend that this test had surprised him. 
G. "I do not have a tablet but I prefer the Two-Finger-Tap to the pinch". This comment is not the only instance in which a similar idea was expressed. Moreover, some of the users who own tablets have also provided the same opinion.

V. "Why did you not implement the Tap\&Tap on tablets too? In my opinion, it is the best in any case. I have never liked the pinch, it is uncomfortable, sometimes it works badly and I am not able to go where I want. The Tap\&Tap, instead, is more precise because it allows the identification of the target area I want to zoom". Undoubtedly, the Tap\&Tap was very appreciated. At any rate, the reasons why it was not tested on tablets was explained. After that, the experimenter asked to try the Tap\&Tap on tablets and the users realized that it was not as advantageous as it seemed.

E. "The Two-Finger-Tap works very well, but I am not sure that it is useful on smartphones because the screen is too small". There were not big doubts regarding the success of the Two-Finger-Tap on tablets. However, these such negative results were not expected on smartphones (other users had also expressed the same).

P. "Even though the Two-Finger-Tap on tablets seems to be faster, the pinch is more amusing". Usually the 'fun effect' decreases over time. Probably, the user does not use the pinch very often and its evaluations are still influenced by the 'fun effect'. At any rate, it indicates that the Two-Finger-Tap (but probably also the Tap\&Tap) is not perceived as amusing.

D. "The Two-Finger-Tap on smartphones does not seem that useful. If I want to zoom deeply, keeping the fingers too close is not possible because, sometimes, the gesture does not work. The pinch is better. On smartphones, instead, the Tap\&Tap is very useful if I have to use the smartphone with just one hand. On the contrary, I continue to prefer the pinch". The user perceives the usefulness of the Tap\&Tap, nevertheless, he prefers the traditional pinch (the comment is not isolated; the pinch is preferred also by other users).

R. "The gestures I tried are useful only if I have to zoom deeply. Otherwise, I think using the traditional pinch is better". The user was deceived by the same expectations rejected and discussed in section 5.3 regarding the execution speed of the gestures when the zoom is deep. At any rate, the user would continue to prefer the traditional pinch.

As seen, the users' opinions are quite different. There are enthusiastic users and more traditional ones who are not willing to change their practices. Anyway, when the users realized that the new gestures were added to the traditional pinch without replacing it, they perceived them as very positive.

\section{Conclusions}

In this paper, two new gestures that enhance the Pinch-Drag-Paradigm were presented. All in all, the evaluations were very positive. The most noteworthy advantage is related to the Tap\&Tap that can be used with just one hand. Both gestures can be implemented easily with few changes in existent software: around 50 lines of code [1] were enough to redesign Leaflet, the base framework used to develop the prototype 
presented in this paper. The implementation of the presented gestures in free and/or commercial software could not be so unfeasible.

Acknowledgments. Thanks to Daniela Bascuñan, linguist, for helping me to translate this paper. Thanks to Giorgio De Michelis and Flavio De Paoli, my PhD supervisors, for their human and academic support. Thanks to Lorenzo Fusco, physicist, for some pieces of advices on the statistical analyses.

\section{References}

1. Bellino, A. (2015, May). Two New Gestures to Zoom: Enhancing Online Maps Services. In Proceedings of the companion publication of the 24rd international conference on World Wide Web companion (future publication). International World Wide Web Conferences Steering Committee.

2. Bier, E.A., Stone, M.C., Pier, K., Buxton, B., and DeRose, T.D. Toolglass and Magic Lenses: The See-Through Interface. In Proc. SIGGRAPH 1993, ACM (1993), 445-446.

3. Boring, S., Ledo, D., Chen, X., Marquardt, N., Tang, A., and Greenberg, S. The fat thumb: using the thumb's contact size for single-handed mobile interaction. In Proc. MobileHCI 2012, ACM (2012), 207-208.

4. Engholm, K.A., and Demory, M.D. "Touch zoom in/out for a graphics display." U.S. Patent No. 6,642,936. 4 Nov. 2003.

5. Fitzmaurice, G.W., Zhai, S., and Chignell, M. Virtual reality for palmtop computers. ACM Trans. Inf. Syst. 11, 3 (1993), 197-218.

6. Hansen, T.R., Eriksson, E., and Lykke-Olesen, A. Mixed interaction space - Expanding the interaction space with mobile devices. In Proc. HCI 2006. Springer London (2006), 365380.

7. Hinckley, K. and Song, H. Sensor synaesthesia: touch in motion, and motion in touch. Proc. CHI, ACM (2011).

8. Hoober, S. How Do Users Really Hold Mobile Devices? UX Matters web site (2013). http://www.uxmatters.com/mt/archives/2013/02/how-do-users-reallyhold-mobiledevices.php, retrieved on 14/01/2015.

9. Malacria, S., Lecolinet, E., and Guiard, Y. Clutch-free panning and integrated pan-zoom control on touch-sensitive surfaces. Proc. CHI, ACM (2010), 2615-2624.

10. Pahud. M., Hinckley, K., Iqbal, S., Sellen, A., and Buxton, B. Toward compound navigation tasks on mobiles via spatial manipulation. In Proc. Mobile HCI 2013. ACM (2013), 113-122.

11. Roth, V., \& Turner, T. (2009, April). Bezel swipe: conflict-free scrolling and multiple selection on mobile touch screen devices. In Proceedings of the SIGCHI Conference on Human Factors in Computing Systems (pp. 1523-1526). ACM.

12. Spelmezan, D., Appert, C., Chapuis, O., and Pietriga, E. Side Pressure for Bidirectional Navigation on Small Devices. In Proc. MobileHCI, ACM (2013), 113-122.

13. Spindler, M., Schuessler, M., Martsch, M., and Dachselt, R. (2014, April). Pinch-dragflick vs. spatial input: rethinking zoom \& pan on mobile displays. In Proceedings of the 32nd annual ACM conference on Human factors in computing systems (pp. 1113-1122). ACM.

14. Wigdor, D., Forlines, C., Baudisch, P., Barnwell, J., and Shen, C. Lucid touch: a seethrough mobile device. In Proc. UIST 2007, ACM (2007), 269-278. 